\title{
Spectral Analysis of the MIXMAX Random Number Generators
}

\author{
Pierre L'Ecuyer \\ Département d'Informatique et de Recherche Opérationnelle, Université de Montréal, Canada, \\ and Inria - Rennes, France, www.iro.umontreal.ca/ lecuyer/ \\ Paul Wambergue \\ École Centrale, Châtenay-Malabray, France, paul.wambergue@gmail.com, \\ Erwan Bourceret \\ École Polytechnique, Palaiseau, France, ebourceret@hotmail.fr,
}

\begin{abstract}
We study the lattice structure of random number generators of the MIXMAX family, a class of matrix linear congruential generators that produce a vector of random numbers at each step. The design of these generators was inspired by Kolmogorov K-systems over the unit torus in the real space, for which the transition function is measure-preserving and produces a chaotic behavior. In actual implementations, however, the state space is a finite set of rational vectors, and the MIXMAX has a lattice structure just like linear congruential and multiple recursive generators. Its matrix entries were also selected in a special way to allow a fast implementation and this has an impact on the lattice structure. We study this lattice structure for vectors of successive and non-successive output values in various dimensions. We show in particular that for coordinates at specific lags not too far apart, in three dimensions, or if we construct points of $k+2$ or more successive values from the beginning of an output vector of size $k$, all the nonzero points lie in only two hyperplanes. This is reminiscent of the behavior of lagged-Fibonacci and AWC/SWB generators. And even if we skip the output coordinates involved in this bad structure, other highly structured projections often remain, depending on the choice of parameters. We show that empirical statistical tests can easily detect this structure.
\end{abstract}

Key words: Random number generators, matrix linear congruential generators, lattice structure, spectral test, simulation

History: This version: October 16, 2018

\section{Introduction}

A matrix linear congruential generator (matrix LCG) of order $k$ with modulus $m$ evolves according to a linear recurrence of the form

$$
\mathbf{x}_{i}=\mathbf{A} \mathbf{x}_{i-1} \bmod m
$$

in which $\mathbf{x}_{i}=\left(x_{i, 0}, \ldots, x_{i, k-1}\right)^{\mathrm{t}}$ is a $k$-dimensional column vector (the ${ }^{\mathrm{t}}$ means transposed) with coordinates in $\mathbb{Z}_{m}=\{0,1, \ldots, m-1\}$, and $\mathbf{A}$ is a $k \times k$ matrix with elements in $\mathbb{Z}_{m}$. The vector $\mathbf{x}_{i} \in \mathbb{Z}_{m}^{k}$ is the state of the generator at step $i$. The total number of possible states is $m^{k}$, and since we must avoid the absorbing state $\mathbf{0}$, the period of the recurrence cannot exceed $m^{k}-1$. This period is attained if and only if $m$ is a prime number and the 
characteristic polynomial of $\mathbf{A}$ is a primitive polynomial modulo $m$ (Niederreiter 1986). The output at step $i$ is the following $k$-dimensional vector of random numbers $u_{i, j} \in[0,1)$ :

$$
\mathbf{u}_{i}=\left(u_{i, 0}, \ldots, u_{i, k-1}\right)=\mathbf{x}_{i} / m \in[0,1)^{k} .
$$

Matrix LCGs have been discussed and studied in Tahmi (1982), Niederreiter (1986), Grothe (1988), and L'Ecuyer (1990, 1994), for example.

The MIXMAX generators are matrix LCGs with special choices of $\mathbf{A}$. They were introduced by Akopov et al. (1991) and Savvidy and Ter-Arutyuntan-Savvidy (1991), and further developed in Savvidy (2015) and Savvidy and Savvidy (2016). The original version proposed by Akopov et al. (1991) had

$$
\mathbf{A}=\mathbf{A}(k, d)=\left(\begin{array}{ccccccc}
1 & 1 & 1 & 1 & \cdots & 1 & 1 \\
1 & 2 & 1 & 1 & \cdots & 1 & 1 \\
1 & 3+d & 2 & 1 & \cdots & 1 & 1 \\
1 & 4 & 3 & 2 & \cdots & 1 & 1 \\
\vdots & & & & \ddots & & \\
1 & k & k-1 & k-2 & \cdots & 3 & 2
\end{array}\right),
$$

in which the integer $d$ in the third row and second column is a parameter that can be chosen in addition to the dimension $k$ and the modulus $m$. We shall call it the MIXMAX- $(m, k, d)$.

The $\mathbf{u}_{i}$ 's also follow the recurrence

$$
\mathbf{u}_{i}=\mathbf{A} \mathbf{u}_{i-1} \bmod 1
$$

which does not involve $m$, and this recurrence is actually defined over the entire unit hypercube $[0,1)^{k} \subset \mathbb{R}^{k}$. When the coordinates of $\mathbf{u}_{0}$ are all rational as in (2), this recurrence necessarily has a finite period, but with irrational coordinates the sequence can be aperiodic and completely fill the space in $[0,1)^{k}$. The original MIXMAX was designed based on an analysis in this aperiodic continuous-space case, and then it was argued that for a large enough $m$, the periodic (discrete) implementation is a very close approximation of the aperiodic (continuous) system. The matrix $\mathbf{A}$ in (3) was selected to satisfy the following two conditions: (1) the determinant of $\mathbf{A}$ is 1 and (2) the eigenvalues $\lambda_{1}, \ldots, \lambda_{k}$ of $\mathbf{A}$ are away from the unit circle. The first condition implies that the linear transformation (4) maps any region $R \subseteq[0,1)^{k}$ into another region of the same volume. The second condition ensures (roughly) that trajectories that start from states very close to each other and evolve according to this transformation in the real space diverge as $\Theta\left(e^{h i}\right)$, where $i$ is the number of steps and $h$ is the Kolmogorov entropy defined as

$$
h=\sum_{j=1}^{k} \mathbb{I}\left[\left|\lambda_{j}\right|>1\right] \cdot \log \left|\lambda_{j}\right|,
$$


in which $\mathbb{I}$ is the indicator function. For more precise statements and further details, see Savvidy and Savvidy (2016). Savvidy (2015) provides lower bounds on $h$ that depend only on $k$ for $\mathbf{A}=\mathbf{A}(k, d)$, and shows that $h$ is reasonably large. In particular, $h$ is much larger than for the AWC and SWB generators of Marsaglia and Zaman (1991). Savvidy (2015) also shows that the maximal period for these generators is $\left(m^{k}-1\right) /(m-1)$, which is $m-1$ times shorter than the maximal possible period for matrix LCGs. This limitation stems from the requirement that $\operatorname{det}(\mathbf{A})=1$. He provides a table of parameters $(k, d)$ for $m=2^{61}-1$ where $k$ ranges from 10 to $3150, d$ ranges from -11 to 15 , and the period is $\left(m^{k}-1\right) /(m-1)$ divided by a small integer (which is 1 in some cases). Even with $k=8$, this already gives a period near $2^{427}$. He finally provides an efficient implementation that uses only $2 k$ additions and one multiplication by $d$ to compute the next vector $\mathbf{x}_{i}$ at each step.

To increase the flexibility and eventually permit a larger entropy and potentially better behavior, Savvidy and Savvidy (2016) defined a MIXMAX variant with an additional integer parameter $c$, with

$$
\mathbf{A}=\mathbf{A}(k, d, c)=\left(\begin{array}{ccccccc}
1 & 1 & 1 & 1 & \ldots & 1 & 1 \\
1 & 2 & 1 & 1 & \ldots & 1 & 1 \\
1 & c+2+d & 2 & 1 & \ldots & 1 & 1 \\
1 & 2 c+2 & c+2 & 2 & \ldots & 1 & 1 \\
1 & 3 c+2 & 2 c+2 & c+2 & \ldots & 1 & 1 \\
& & & \ldots & & & \\
1 & (k-2) c+2 & (k-3) c+2 & (k-4) c+2 & \cdots & c+2 & 2
\end{array}\right),
$$

which we call MIXMAX- $(m, k, d, c)$, and another variant with five parameters $(m, k, d, c, b)$, with

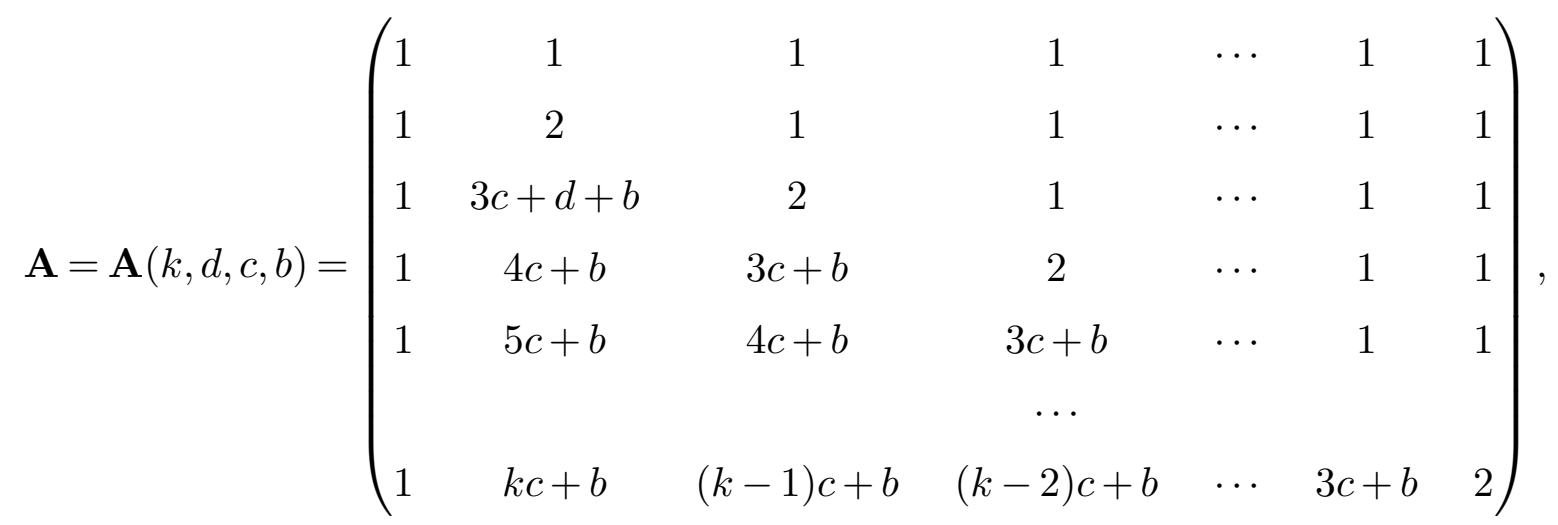

which we denote by MIXMAX- $(m, k, d, c, b)$. These generators also satisfy the conditions (1) and (2) above and have the same maximal possible period as the MIXMAX- $(m, k, d)$. Note that in those papers, the parameters $m, k, d, c$ are named $p, N, s, m$ instead. A user's guide with specific parameters and pointers to downloadable code is available in Savvidy (2017). This code is part of the ROOT library available at the CERN, in Geneva. 
Examining the eigenvalues of $\mathbf{A}$ and the entropy $h$ is one type of spectral analysis for matrix LCGs. A different type of spectral analysis is the spectral test that examines the lattice structure of vectors of successive or non-successive output values produced by LCGs (Coveyou and MacPherson 1967, Knuth 1998, L'Ecuyer and Couture 1997). The purpose of this paper is to show how this spectral test applies to MIXMAX generators and see what kind of lattice structure we can find.

The remainder is organized as follows. In Section 2, we recall the lattice structure of matrix LCGs and how the spectral test works for these generators. In Section 3, we prove various properties of the lattice structure of the MIXMAX generators. We show that certain coordinates of the successive output points are linked by very simple linear relations, which implies that these points belong to a small number of parallel hyperplanes in the unit hypercube. In Section 4, we show that undesirable structures remain even if we skip certain output values to break the most problematic linear relations. In Section 5, we illustrate this with specific MIXMAX generators proposed in Savvidy (2017). What we find is reminiscent of the types of bad structures found in the Lagged-Fibonacci, AWC/SWB, and some other types of multiple recursive generators (Tezuka et al. 1993, L'Ecuyer 1997, L'Ecuyer and Touzin 2004, L'Ecuyer and Simard 2014).

For general background on uniform random number generators (RNGs), we refer the reader to the tutorial chapter of L'Ecuyer (2012), the recent survey of L'Ecuyer et al. (2017), and the detailed historical account of L'Ecuyer (2017).

\section{Lattice Structure of Matrix LCGs}

Suppose a matrix LCG produces $s$ uniform random numbers $u_{0}, \ldots, u_{s-1}$ as follows. Starting from some initial state $\mathbf{x}_{0}$, we generate $\mathbf{u}_{0}, \ldots, \mathbf{u}_{\nu}$ as in (2) where $\nu$ satisfies $s=k \nu+r$ and $0 \leq r<k$, and we put $u_{k i+j}=u_{i, j}$ for all $i=0, \ldots, \nu$ and $j=0, \ldots, k-1$ for which $0 \leq k i+j<s$. Let $\Psi_{s}$ be the set of all vectors $\left(u_{0}, \ldots, u_{s-1}\right)$ obtained in this way, from all the $m^{k}$ possible initial states $\mathbf{x}_{0}$ of the matrix LCG, including the zero vector. In case the generator does not have full period $m^{k}-1$, the set $\Psi_{s}$ considered here contains all vectors produced over all cycles of the generator. An important requirement of good RNGs is that this set $\Psi_{s}$ covers the unit hypercube $[0,1)^{s}$ very evenly, at least when $s$ is not too large (L'Ecuyer 1994, 2006). This requirement captures uniformity and independence of the successive random numbers; that is, it measures the quality of the approximation of the theoretical concept of independent uniform random variables (Knuth 1998, L'Ecuyer 2006). Note that for $s=k, \Psi_{s}$ contains all the $m^{k}$ points of $\mathbb{Z}_{m}^{k} / m=\{0,1 / m, \ldots,(m-1) / m\}^{k}$

exactly once. For $s<k, \Psi_{s}$ is a multiset that contains all points $\mathbb{Z}_{m}^{s} / m$ exactly $m^{k-s}$ times each. This is the best that one can achieve, given $m$ and $k$.

We also consider the following generalized form of this notion, as in Couture and L'Ecuyer (1994), L'Ecuyer (1997), L'Ecuyer and Couture (1997), and L'Ecuyer and Simard (2014). For any finite set of integers $I=\left\{i_{1}, \ldots, i_{s}\right\}$ where $0 \leq i_{1}<\cdots<i_{s}$, consider the multiset 
$\Psi_{s}(I)$ of all $s$-dimensional output vectors $\left(u_{i_{1}}, \ldots, u_{i_{s}}\right)$ obtained by the method described earlier, from all possible initial states $\mathbf{x}_{0}$ :

$$
\Psi_{s}(I)=\left\{\left(u_{i_{1}}, \ldots, u_{i_{s}}\right) \in[0,1)^{s} \mid \mathbf{x}_{0} \in \mathbb{Z}_{m}^{k}\right\}
$$

If $\mathbf{x}_{0}$ is selected randomly and uniformly from $\mathbb{Z}_{m}^{k}$, then $\left(u_{i_{1}}, \ldots, u_{i_{s}}\right)$ has the uniform distribution over the finite multiset $\Psi_{s}(I)$. The ideal, on the other hand, would be that this vector has the uniform distribution over $[0,1)^{s}$. Obviously, we can have a good approximation of this ideal only if $\Psi_{s}(I)$ covers $[0,1)^{s}$ very evenly, or at least not too unevenly. This multiset $\Psi_{s}(I)$ is actually the projection of the set $\Psi_{s^{\prime}}$ over the selected coordinate indices $i_{1}, \ldots, i_{s}$, with $s^{\prime}=i_{s}+1$. The set $\Psi_{s}$ is just a special case of this with $I=\{0, \ldots, s-1\}$. For $s<k$, it is not always true that $\Psi_{s}(I)$ contains all of $\mathbb{Z}_{m}^{s} / m$; for some sets $I$ it can contain only a small proportion.

It is known that any projection $\Psi_{s}(I)$ of some $\Psi_{s^{\prime}}$ over a subset of $s$ coordinates, is the (finite) intersection of a lattice $L_{s}(I)$ in the real space $\mathbb{R}^{s}$ with the unit hypercube $[0,1)^{s}$ (Afflerbach and Grothe 1988, L'Ecuyer and Couture 1997). One consequence of this is that there are families of equidistant parallel hyperplanes in $\mathbb{R}^{s}$ such that each family contains all the points of $\Psi_{s}(I)$. We want to make sure that none of these families has just a few widelyspaced hyperplanes, because this would imply that the points would not cover the space very well. The spectral test introduced by Coveyou and MacPherson (1967) for LCGs and further discussed in Knuth (1998) and L'Ecuyer and Couture (1997), for example, computes the distance $d_{s}(I)$ between successive hyperplanes for the family for which this distance is largest. Let $n=\min \left(m^{k}, m^{s}\right)$, which is the largest number of distinct points that we can have in $\Psi_{s}(I)$. A standardized measure that takes values in $(0,1]$ regardless of $n$ and $s$ can be defined as $S_{s}(I)=d_{s}^{*}(n) / d_{s}(I)$, where the normalizing constant $d_{s}^{*}(n)$ is the smallest distance between hyperplanes that can be achieved by a general $s$-dimensional lattice having $n$ points per unit of volume. One can write $d_{s}^{*}(n)=\gamma_{s}^{1 / 2} n^{-1 / s}$ where the $\gamma_{s}$ are the Hermite constants, which depend only on $s$ (Conway and Sloane 1999, L'Ecuyer 1999). These constants are known exactly only for $s \leq 8$ and $s=24$. For the other values of $s$, we replace $d_{s}^{*}(n)$ in the definition of $S_{s}(I)$ by the value of $d_{s}(I)$ achieved by the best known lattice of density $n$ in $s$ dimensions. In other words, we approximate $\gamma_{s}^{1 / 2}$ by the smallest value of $n^{1 / s} d_{s}(I)$ that has been achieved by a known lattice construction in $s$ dimensions. These values can be found in Chapter 6 of Conway and Sloane (1999). We need them only for $s<20$. Thus, $S_{s}(I)$ can be arbitrarily close to 1 in general. Very small values of $S_{s}(I)$ are bad and should be avoided.

It is known that $d_{s}(I)=1 / \ell_{s}(I)$ where $\ell_{s}(I)$ is the (Euclidean) length of the shortest nonzero vector in the dual lattice

$$
L_{s}^{*}(I)=\left\{\mathbf{w} \in \mathbb{R}^{s}: \mathbf{w}^{\mathrm{t}} \mathbf{v} \bmod 1=0 \text { for all } \mathbf{v} \in L_{s}(I)\right\}
$$

To find a shortest vector, one first constructs a basis of the dual lattice. Then a shortest vector and its length are a solution and the optimal value of a quadratic integer optimization 
problem that can be solved by a branch-and-bound procedure (Fincke and Pohst 1985, L'Ecuyer and Couture 1997).

If we compute a shortest nonzero vector in the dual lattice with the $L^{1}$ norm defined by $\|\mathbf{w}\|_{1}=\left\|\left(w_{1}, \ldots, w_{s}\right)\right\|_{1}=\left|w_{1}\right|+\cdots+\left|w_{s}\right|$ instead of the Euclidean norm, the length of the shortest vector minus one gives the minimal number of hyperplanes that cover all the lattice points in $[0,1)^{s}$ (Marsaglia 1968, Knuth 1998).

The lattice bases can be constructed as follows. Suppose $s=k \nu+r$ and for any $k \times k$ matrix $\mathbf{M}$, let $[\mathbf{M}]_{r}$ denote the $r \times k$ matrix formed by the first $r$ rows of $\mathbf{M}$. Let $\mathbf{I}$ be the identity matrix. Consider the $s \times s$ matrices

$$
\mathbf{V}=\left(\begin{array}{ccccc}
\mathbf{I} / m & \mathbf{A}^{\mathrm{t}} / m & \cdots & \left(\mathbf{A}^{\nu-1}\right)^{\mathrm{t}} / m & \left(\left[\mathbf{A}^{\nu}\right]_{r}\right)^{\mathrm{t}} / m \\
\mathbf{0} & \mathbf{I} & & & \mathbf{0} \\
& & \ddots & & \\
\mathbf{0} & & & \mathbf{I} & \mathbf{I}
\end{array}\right)
$$

and

$$
\mathbf{W}=\left(\begin{array}{cccccc}
m \cdot \mathbf{I} & \mathbf{0} & & & \mathbf{0} & \\
-\mathbf{A} & \mathbf{I} & & & & \\
\vdots & & \ddots & & & \\
-\mathbf{A}^{\nu-1} & & & \mathbf{I} & & \\
-\left[\mathbf{A}^{\nu}\right]_{r} & \mathbf{0} & & & & \mathbf{I}
\end{array}\right)
$$

in which the identity $\mathbf{I}$ at the bottom right of each matrix is $r \times r$ and the other $\mathbf{I}$ 's are $k \times k$. Then the rows of $\mathbf{V}$ form a basis of the lattice $L_{s}$ (Afflerbach and Grothe 1988) and, since $\mathbf{V W}^{\mathrm{t}}=\mathbf{I}$, the rows of $\mathbf{W}$ form the corresponding dual basis, which is a basis of the dual lattice $L_{s}^{*}$. Any integer linear combination of the rows of $\mathbf{W}$ belongs to the dual lattice.

For the case of lacunary indices, a set of generating vectors for $L_{s}(I)$ can be obtained by projecting the basis vectors of $L_{s^{\prime}}$ over the $s$ retained coordinates in $I$. That is, we build $\mathbf{V}$ with $s^{\prime}$ columns and then we keep only the columns of $\mathbf{V}$ whose indices are in $I$, and discard the other ones. The $s^{\prime}$ rows of the resulting matrix give a set of $s^{\prime}$ vectors that can be turned into a set of $s$ independent vectors that form a basis of $L_{s}(I)$, using the approach described in L'Ecuyer and Couture (1997). The corresponding dual basis can then be obtained by inverting this basis matrix modulo 1 . (Note that projecting the vectors of the original dual basis on the retained lacunary coordinates does not provide vectors that belong to the dual basis of $L_{s}(I)$ in general.)

Ideally, we want to make sure that no $S_{s}(I)$ is very small, at least for a selected class of subsets $I$. We cannot verify this for all possible subsets $I$ of arbitrary size, because there are too many. The idea is to limit ourselves to a finite class of subsets $I$ and make sure that 
the smallest $S_{s}(I)$ (the worst-case) over that class is not too small. One way to define this class is to select an integer $s^{\prime}>0$ and consider all subsets $I \subseteq\left\{0, \ldots, s^{\prime}-1\right\}$. This gives the worst-case figure of merit

$$
M_{s^{\prime}}=\min _{I \subseteq\left\{0, \ldots, s^{\prime}-1\right\}} S_{s}(I)
$$

which we will use in the rest of this paper. We will always take $s^{\prime}>k$, because for $s^{\prime} \leq k$ all the projections contain all the points of $\mathbb{Z}_{m}^{s} / m$. If $s^{\prime}$ is taken very large, for example $s^{\prime}>100$, it may be impossible to have $M_{s^{\prime}}$ very close to 1 , because an excessively large number of subsets $I$ are considered and it may be impossible to have $S_{s}(I)$ close to 1 for all of them simultaneously. We do not know what is the best achievable $M_{s^{\prime}}$ as a function of $s^{\prime}$. But empirically, we find that for large $m$ and moderate $k$, with random choices of the matrix A, it is rare to find a matrix LCG with a very small value of $M_{s^{\prime}}$. The following example illustrates this situation.

EXAMPLE 1. Consider a multiple recursive generator (MRG), defined via:

$$
x_{i}=\left(a_{1} x_{i-1}+\cdots+a_{k} x_{i-k}\right) \bmod m, \quad u_{i}=x_{i} / m,
$$

which is in fact equivalent to a matrix LCG of order $k$ with matrix

$$
\mathbf{A}=\left(\begin{array}{cccc}
0 & 1 & \ldots & 0 \\
\vdots & & \ddots & \vdots \\
0 & 0 & \ldots & 1 \\
a_{k} & a_{k-1} & \cdots & a_{1}
\end{array}\right)^{k}
$$

For $k=7$ and $m=2^{63}-52425$ (a prime), we made a random search for 10,000 parameter vectors $\mathbf{a}=\left(a_{1}, \ldots, a_{k}\right)$ that give a full period $\rho=m^{k}-1 \approx 2^{441}$, and we then computed $M_{10}$ and $M_{12}$ for each. Their values of $M_{10}$ ranged from 0.53858 (the best) to 0.13221 (the worst), and $M_{12}$ ranged from 0.40807 to 0.10540 . Clearly, values of $M_{10}$ or $M_{12}$ smaller than say 0.1 are very rare. In Section 5, we will find that the corresponding values for the MIXMAX are several orders of magnitude smaller.

Although computing $S_{s}(I)$ for a large class of sets $I$ can be expensive, by looking at the structure of the generator, one can sometimes identify specific subsets $I$ for which $S_{s}(I)$ is very small for some $s^{\prime}$ not too large, due to an unfortunate design. This is what we do for the MIXMAX in this paper. For excellent generators, such systematic structural defects should not happen. Note that for any two sets $I \subset I^{\prime}$ of sizes $s<s^{\prime}$, any vector in $L_{s}^{*}(I)$ can be extended to a vector of the same length in $L_{s^{\prime}}^{*}\left(I^{\prime}\right)$ by adding zero coordinates. Therefore $\ell_{s^{\prime}}\left(I^{\prime}\right) \leq \ell_{s}(I)$, or equivalently $d_{s}(I) \leq d_{s^{\prime}}\left(I^{\prime}\right)$. This means that if $d_{s}(I)$ is large for some set $I$ of small cardinality $s$, then $d_{s^{\prime}}\left(I^{\prime}\right)$ must be at least as large for all sets $I^{\prime}$ that contain $I$. For this reason, when $S_{s}(I)$ is extremely small, it is often the case that $d_{s^{\prime}}\left(I^{\prime}\right)=d_{s}(I)$ for a very large number of supersets $I^{\prime}$ that contain $I$, and $S_{s^{\prime}}\left(I^{\prime}\right)$ will typically be also very small 
for most of them. Conversely, this same inequality implies that if $d_{s^{\prime}}\left(I^{\prime}\right)$ is large for some set $I^{\prime}$, then for any set $I \subset I^{\prime}$, it cannot be small.

There is one important difference between the lattice structure of matrix LCGs and that of ordinary LCGs and MRGs. In the latter, the point set $\Psi_{s}(I)$ and its lattice structure are shift-invariant with respect to coordinate numbers, in the sense that $\Psi_{s}(I)$ for $I=\left\{i_{1}, \ldots, i_{s}\right\}$ is the same as $\Psi_{s}\left(I^{\prime}\right)$ for $I^{\prime}=\left\{i_{1}+j, \ldots, i_{s}+j\right\}$ for any integer $j \geq 0$, whereas for the matrix LCG, apart from special cases, this is true only if $j$ is a multiple of $k$. That is, the lattice structure we analyze is for points whose coordinates are numbered from the beginning of an output vector.

\section{Lattice Structure of MIXMAX}

When a MIXMAX generator has maximal period, it has $m-1$ cycles of length $\left(m^{k}-\right.$ $1) /(m-1)$, and the initial state determines which cycle we are in. In this paper, we study the lattice $L_{s}$ or $L_{s}(I)$ generated by all the points produced over all the cycles of the MIXMAX generator. When a generator has many disjoint cycles, one cannot rule out a priori that the lattice generated by a single cycle is a strict sublattice of the full lattice generated by all the points produced over all the cycles. Analyzing the lattice generated over a single cycle is beyond the scope of the present paper. It could be done using the ideas and methodology of Couture and L'Ecuyer (1996).

By writing the matrix $-\mathbf{A}=-\mathbf{A}(k, d)$ explicitly in rows $k+1$ to $2 k$ of the definition of $\mathbf{W}$, we find that for the MIXMAX- $(m, k, d)$,

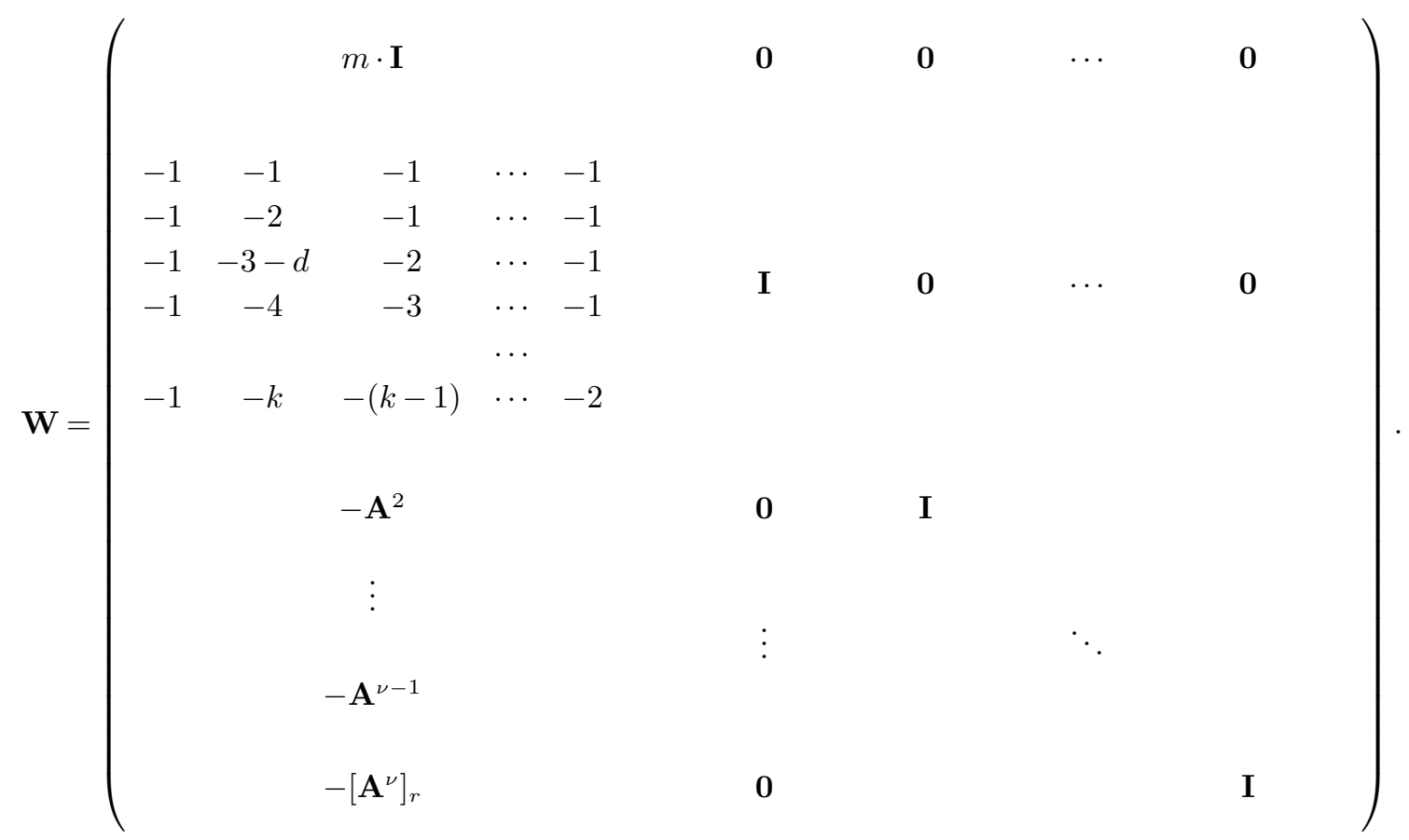

For the MIXMAX- $(m, k, d, c)$ and $\operatorname{MIXMAX}-(m, k, d, c, b)$, it suffices to replace the explicit submatrix $-\mathbf{A}=-\mathbf{A}(k, d)$ in $\mathbf{W}$ by $-\mathbf{A}(k, d, c)$ and $-\mathbf{A}(k, d, c, b)$, respectively. The rows 
of $\mathbf{W}$, which we denote by $\mathbf{w}_{1}, \ldots, \mathbf{w}_{s}$, form a basis of the dual lattice, so any integer linear combination of these vectors belongs to the dual lattice $L_{s}^{*}$.

In particular, if $k \geq 2$, we have

$$
\begin{aligned}
\mathbf{w}_{k+1}= & (-1,-1,-1, \ldots,-1,1,0, \ldots, 0) \\
\mathbf{w}_{k+2}= & (-1,-2,-1, \ldots,-1,0,1, \ldots, 0) \\
& \uparrow \text { coordinate } k+1
\end{aligned}
$$

and therefore

$$
\begin{array}{r}
\mathbf{w}=\mathbf{w}_{k+1}-\mathbf{w}_{k+2}=(0,1,0, \ldots, 0,1,-1,0, \ldots, 0) \\
\uparrow \text { coordinate } k+1
\end{array}
$$

is in the dual lattice $L_{s}^{*}$ for any $s \geq k+2$. (Here, the index $i$ of output values and the coordinates of $\mathbf{u}_{i}$ start at 0 as usual, but we start the coordinates of vectors $\mathbf{v}$ and $\mathbf{w}$ at 1 , which is also standard.) This vector $\mathbf{w}$ has Euclidean length $\sqrt{3}$ and $L^{1}$ norm equal to 3 . Its presence in the dual lattice implies that if we take all $k$ output values at each step, the successive output values satisfy $\left(u_{1}+u_{k}-u_{k+1}\right) \bmod 1=0$. And since $0 \leq u_{i}<1$ for all $i$, one must have $u_{1}+u_{k}-u_{k+1}=q$ for $q \in\{0,1\}$. This means that if we take $I=\{1, k, k+1\}$, all the points of $\Psi_{3}(I)$ are in only two planes, determined by this equation. Also, the dual lattice to $L_{3}(I)$ contains the vector $(1,1,-1)$, whose Euclidean length is $\sqrt{3}$. This argument holds in exactly the same way for the MIXMAX- $(m, k, d, c)$ and MIXMAX- $(m, k, d, c, b)$ as well, because they have the same first two rows of $\mathbf{A}$. We have just proved the following.

Proposition 1. For the three MIXMAX variants, with $k \geq 2$, whenever $I=\{1, k, k+$ $1\} \subseteq I^{\prime}$, all the points of $\Psi_{3}\left(I^{\prime}\right)$ are in two equidistant parallel planes, which are at distance $1 / \sqrt{3}$ apart, in the three dimensional unit hypercube.

This result shows not only that the lattice structure is very bad for $\Psi_{3}(I)$, but also that it is bad for a very large number of projections on subsets $I^{\prime}$ of coordinates that contain $I$. This is the same type of problematic structure as for the lagged-Fibonacci and AWC/SWB generators (Couture and L'Ecuyer 1994, L'Ecuyer 1997, Tezuka and L'Ecuyer 1992, Tezuka et al. 1993), which are known to fail simple statistical tests because of this structure (L'Ecuyer and Simard 2007). We will see in Section 5 that the MIXMAX also fails simple tests because of this structure.

The next proposition unveils another structure of the $\operatorname{MIXMAX-}(m, k, d)$. If $k \geq 5$, by taking

$$
\begin{gathered}
\mathbf{w}=\mathbf{w}_{2 k}-\mathbf{w}_{2 k-1}-\mathbf{w}_{k+1}=(1,0, \ldots, 0,-1,0, \ldots, 0,-1,1,0, \ldots, 0) \\
\uparrow \text { coord } k+1
\end{gathered}
$$

in which we have -1 at coordinates $k+1$ and $2 k-1$, we find with a similar reasoning: 
Proposition 2. For the MIXMAX- $(m, k, d)$ with $k \geq 5$, if $\{0, k, 2 k-2,2 k-1\} \subseteq I$, then all the points of $\Psi_{s}(I)$ are in the three equidistant parallel planes with equations $u_{0}-u_{k}-$ $u_{2 k-2}+u_{2 k-1}=q$ for $q \in\{-1,0,1\}$, which are at distance $1 / 2$ apart.

Note that in contrast to the previous one, this relationship does not involve the second coordinate of the state vectors of size $k$. One can find other undesirable relationships like this and we will give a few more in what follows.

\section{Skipping Coordinates}

The simplest way to eliminate the bad structure exhibited in the previous propositions is to skip some coordinates of the $k$-dimensional vector $\mathbf{u}_{i}$ when producing the output. This idea was already proposed by Lüscher (1994) for the AWC/SWB generators and implemented by James (1994). Instead of taking all $k$ coordinates at each step, one can retain only a subset $J \subset\{0, \ldots, k-1\}$ of the $k$ coordinates, to produce a block of $|J|$ random numbers at each step. For example, if we skip the second coordinate of each vector, i.e., if we take $J=\{0,2,3, \ldots, k-1\}$, the relationship $u_{1}+u_{k}-u_{k+1}=0$ or 1 is not harmful anymore, because $u_{1}$ and $u_{k+1}$ are no longer used. But other relationships can be found that do not involve these removed coordinates (Proposition 2 gives one), and some of these relationships may correspond to short vectors in the dual of the corresponding projected lattice $L_{s}(I)$, where $I$ contains only coordinates that are retained. To find such a relationship, we need to find a vector $\mathbf{w} \in L_{s}^{*}$ whose coordinates that correspond to the output values that are skipped are zero. (In the notation used in this paper, the indices of the $u_{i}$ 's when we skip coordinates remain the same as when we take all $k$ values at each step; we find this less confusing than making them depend on $I$ or $J$.)

If the set $J$ of coordinates that we keep includes the first two coordinates, then the relationship $u_{1}+u_{k}-u_{k+1}=0$ or 1 still involves coordinates that are all retained and the bad lattice structure remains. To remove this structure, one must skip at least one of the first two coordinates. For the MIXMAX- $(m, k, d)$, if we skip only the second coordinate of each $\mathbf{u}_{i}$, the problem remains, because the relationship in Proposition 2 does not involve $u_{1}, u_{k+1}, u_{2 k+1}, \ldots$, so we still have the bad hyperplane structure pointed out in this proposition.

The next proposition shows that even if we skip the first two (and even the first three) coordinates of each $\mathbf{u}_{i}$, for the MIXMAX- $(m, k, d)$ we still get bad relationships among the other coordinates, and therefore a bad structure. In all our propositions, the bad structure also holds for all sets $I^{\prime}$ that contain $I$.

Proposition 3. For the MIXMAX- $(m, k, d)$, if $k \geq 6,0 \leq j \leq k-6$, and $\{5+j, k+$ $3+j, k+4+j, k+5+j\} \subseteq I$, then $\Psi_{s}(I)$ is contained in at most 4 equidistant parallel hyperplanes at distance $1 / \sqrt{7}$ apart. If $k \geq 7,0 \leq j \leq k-7$, and the stronger condition $\{5+j, 6+j, k+3+j, k+4+j, k+5+j, k+6+j\} \subseteq I$ holds, then $\Psi_{s}(I)$ is also contained in 
5 equidistant parallel hyperplanes at distance $1 / \sqrt{6}$ apart. These 5 hyperplanes are not the same as the 4 hyperplanes in the first part.

Proof. For the first part, take

$$
\begin{gathered}
\mathbf{w}=\mathbf{w}_{k+4+j}-2 \mathbf{w}_{k+5+j}+\mathbf{w}_{k+6+j}=(0, \ldots, 0,-1,0, \ldots, 0,1,-2,1,0, \ldots) \\
\uparrow \operatorname{coord.~} 6+j
\end{gathered}
$$

in which the -1 is at position $6+j$ and the -2 is at position $k+5+j$. This shows that we have the relationship $-u_{5+j}+u_{k+3+j}-2 u_{k+4+j}+u_{k+5+j}=q$ for $q \in\{-2,-1,0,1\}$. These are the equations of four hyperplanes that contain all the points of $\Psi_{s}(I)$ when $I$ contains the coordinates involved in this linear relationship. In this case, the lattice $L_{s}(I)$ contains a dual vector with $L^{1}$-norm of 5 and Euclidean length $\sqrt{7}$.

For the second part, take

$$
\begin{gathered}
\mathbf{w}=\mathbf{w}_{k+4+j}-\mathbf{w}_{k+5+j}-\mathbf{w}_{k+6+j}+\mathbf{w}_{k+7+j}=(0, \ldots, 0,-1,-1,0, \ldots, 0,1,-1,-1,1,0, \ldots) . \\
\uparrow \text { coord. } 6+j
\end{gathered}
$$

in which the first -1 is at position $6+j$ and the first 1 is at position $k+4+j$. This dual basis vector indicates the relationship $-u_{5+j}-u_{6+j}+u_{k+3+j}-u_{k+4+j}-u_{k+5+j}+u_{k+6+j}=q$ for $q \in\{-3,-2,-1,0,1\}$, which involves only retained coordinates if $I$ satisfies the condition in the second part of the proposition. Then the lattice $L_{s}(I)$ contains a dual vector with $L^{1}$-norm of 6 and Euclidean length $\sqrt{6}$, and the conclusion follows. Since the condition of the second part implies that of the first part, the result of the first part also holds here. The two sets of hyperplanes are different.

By taking for $\mathbf{w}$ the same combination of dual vectors as in the first part of the previous proposition, we obtain the following two results for the MIXMAX with four and five parameters. In all the propositions that follow, $\ell$ will denote the length of a short vector in the dual lattice, but not necessarily the shortest length.

Proposition 4. For the MIXMAX- $(m, k, d, c)$ generator with $c \geq 1$, if $k \geq 6,0 \leq j \leq$ $k-6$, and $\{4+j, 5+j, k+3+j, k+4+j, k+5+j\} \subseteq I$, then there is a set of $c+3$ equidistant parallel hyperplanes that contain all the points of $\Psi_{s}(I)$. These hyperplanes are at distance $1 / \ell$ apart, where $\ell^{2}=(c-1)^{2}+7$.

Proof. The dual basis contains the vector

$$
\begin{gathered}
\mathbf{w}=\mathbf{w}_{k+4+j}-2 \mathbf{w}_{k+5+j}+\mathbf{w}_{k+6+j}=(0, \ldots, 0,1-c,-1,0, \ldots, 0,1,-2,1,0, \ldots), \\
\uparrow \text { coord. } 5+j
\end{gathered}
$$

in which the $1-c$ is at position $5+j$ and the -2 is at position $k+5+j$. Thus we have the relationship $(1-c) u_{4+j}-u_{5+j}+u_{k+3+j}-2 u_{k+4+j}+u_{k+5+j}=q$ where $q \in\{-c-1,-c, \ldots, 0,1\}$. Also, the square Euclidean length of $\mathbf{w}$ is $(c-1)^{2}+7$. The result follows. 
Proposition 5. For the MIXMAX- $(m, k, d, c, b)$ generator with $c \geq 1$ and $b \geq 0$, if $k \geq 6$, $0 \leq j \leq k-6$, and $\{3+j, 4+j, 5+j, k+3+j, k+4+j, k+5+j\} \subseteq I$, then there is a set of $5 c+2 b-1$ equidistant parallel hyperplanes that contain all the points of $\Psi_{s}(I)$. These hyperplanes are at distance $1 / \ell$ apart, where $\ell^{2}=(2 c+b-2)^{2}+(3 c+b-3)^{2}+7$.

Proof. The dual lattice contains

$$
\begin{gathered}
\mathbf{w}=\mathbf{w}_{k+4+j}-2 \mathbf{w}_{k+5+j}+\mathbf{w}_{k+6+j}=(0, \ldots, 0,2 c+b-2,-3 c-b+3,-1,0, \ldots, 0,1,-2,1,0, \ldots) \\
\uparrow \operatorname{coord} .4+j
\end{gathered}
$$

in which the -2 is at position $k+5+j$. Thus, $\left((2 c+b-2) u_{3+j}-(3 c+b-3) u_{4+j}-u_{5+j}+\right.$ $\left.u_{k+3+j}-2 u_{k+4+j}+u_{k+5+j}\right) \bmod 1=0$. If $I$ contains the coordinates involved in this linear relationship, the lattice $L_{s}(I)$ contains a dual vector with $L^{1}$-norm of $5 c+2 b$ and square Euclidean length $(2 c+b-2)^{2}+(3 c+b-3)^{2}+7$.

Propositions 4 and 5 tell us that the $\operatorname{MIXMAX}-(m, k, d, c)$ has a bad structure when $c$ is small, and the MIXMAX- $(m, k, d, c, b)$ has a bad structure when both $c$ and $b$ are close to 0 , respectively. The next proposition emphasizes the fact that a large value of $c$ is not sufficient to guarantee good quality. It shows that if the modulus $m$ is near a small multiple of $c$, there is also a bad structure.

Proposition 6. For the MIXMAX-(m,k,d,c) generator with $c \geq 1$, suppose $m=q c+r$ where $q>0$ and $|r|$ are small integers ( $r$ can be negative).

If $k \geq 6,0 \leq j \leq k-6$, and $\{4+j, 5+j, k+3+j, k+4+j, k+5+j\} \subseteq I$, then there is a set of $5 q+|q+r|-1$ equidistant parallel hyperplanes that contain all the points of $\Psi_{s}(I)$. These hyperplanes are at distance $1 / \ell$ apart, where $\ell^{2}=7 q^{2}+(q+r)^{2}$.

Under the stronger conditions that $k \geq 7,0 \leq j \leq k-7$, and $\{4+j, 5+j, 6+j, k+3+$ $j, k+4+j, k+5+j, k+6+j\} \subseteq I, \Psi_{s}(I)$ is covered by another family of equidistant parallel hyperplanes at distance $1 / \ell$ apart, where $\ell^{2}=5 q^{2}+r^{2}+(q+r)^{2}$. This last bound is smaller than the bound in the first part if and only if $2 q^{2}>r^{2}$.

In both cases, if $d=0$, one can also take $j=-1$ and reduce the lower bound on $k$ by 1 .

Proof. For the first part, take

$$
\begin{aligned}
\mathbf{w} & =q\left(\mathbf{w}_{k+4+j}-2 \mathbf{w}_{k+5+j}+\mathbf{w}_{k+6+j}\right) \bmod m \\
& =q(0, \ldots, 0,1-c,-1,0, \ldots, 0,1,-2,1,0, \ldots) \bmod m \\
& =(0, \ldots, 0, q+r,-q, 0, \ldots, 0, q,-2 q, q, 0, \ldots)
\end{aligned}
$$

in which the $q+r$ is at position $5+j$ and the $-2 q$ is at position $k+5+j$. Thus we have the relationship $\left((q+r) u_{4+j}-q u_{5+j}+q u_{k+3+j}-2 q u_{k+4+j}+q u_{k+5+j}\right) \bmod 1=0$. So if $I$ satisfies the condition, we have a dual vector whose $L^{1}$ length at most $5 q+|q+r|$ and squared Euclidean length $7 q^{2}+(q+r)^{2}$. 
For the second part, we have

$$
\begin{aligned}
\mathbf{w} & =q\left(\mathbf{w}_{k+4+j}-\mathbf{w}_{k+5+j}-\mathbf{w}_{k+6+j}+\mathbf{w}_{k+7+j}\right) \bmod m \\
& =q(0, \ldots, 0,1-c,-c,-1,0, \ldots, 0,1,-1,-1,1,0, \ldots) \bmod m \\
& =(0, \ldots, 0, q+r, r,-q, 0, \ldots, 0, q,-q,-q, q, 0, \ldots),
\end{aligned}
$$

in which the $q+r$ is at position $5+j$ and the first $q$ is at position $k+4+j$. If $I$ satisfies the condition in the first part, we have a vector in the dual of $L_{s}(I)$ whose only nonzero coordinates correspond to the nonzero coordinates of $\mathbf{w}$. This vector has $L^{1}$ length of $5 q+$ $|r|+|q+r|$ and squared Euclidean length $5 q^{2}+r^{2}+(q+r)^{2}$.

When $d=0$, one can easily verify that this development also works for $j=-1$.

We have seen so far that the MIXMAX always produces a bad lattice structure if we keep the first two coordinates of each vector. If we skip these two coordinates, there is still always a bad lattice structure for the MIXMAX- $(m, k, d)$, as seen in Proposition 3 , and for the MIXMAX with four or five parameters, the lattice structure is always bad if the parameters $c$ and $b$ are too small (e.g., if $b=0$ and $c$ is close to a divisor of $m$ ). Moreover, even if $c$ is large and we skip the first three values of each vector, there are situations where the lattice structure is also bad, depending on the choices of parameters $c$ and $b$. In other situations, the lattice structure can be explored by applying the spectral test numerically to specific MIXMAX instances. That is, for $s=k+1, k+2, \ldots$, we construct a basis of the dual lattice for $I=\{0, \ldots, s-1\}$ as explained in Section 3, then we compute the length $\ell_{s}$ of the shortest nonzero vector in that lattice using the methodology described in L'Ecuyer and Couture (1997).

\section{Testing of some proposed MIXMAX generators}

We now apply our results to specific MIXMAX generators proposed by Savvidy (2017). We also give empirical results for two simple empirical statistical tests that detect the bad structures, namely the collision test and the birthday spacings tests. These tests are studied in details in L'Ecuyer and Simard (2001) and L'Ecuyer et al. (2002); here we just summarize their definitions. For the collision test, we split the interval $[0,1)$ into $d$ equal parts. This partitions the unit cube $[0,1)^{s}$ into $d^{s}$ cubic boxes. Then we generate $n$ random vectors in $s$ dimensions by taking $n$ non-overlapping blocks of $s$ successive output values produced by the generator, and we count the number $C$ of times a point falls in a box that already had a point before (the number of collisions). We repeat this $N$ times independently and let $C_{\text {tot }}$ be the total number of collisions (the sum of the $N$ realizations of $C$ ). This $C_{\text {tot }}$ should have approximately a Poisson distribution with mean $\lambda_{c}=N n^{2} /\left(2 d^{s}\right)$. We compute and report the right $p$-value, defined as the probability that a Poisson random variable with mean $\lambda_{c}$ takes a value larger or equal to the observed realization of $C_{\text {tot }}$. A very small $p$ -

value indicates that the points tend to fall in the same boxes (or same regions in the unit 
cube) much more often that they should. The birthday spacings test is similar, except that we number the $d^{s}$ boxes in some natural order, sort the box numbers of the $n$ points by increasing order, compute the first-order differences between the successive box numbers, and count the number of collisions in these differences. The total number of collisions $B_{\text {tot }}$ over the $N$ replications should be approximately Poisson with mean $\lambda_{b}=N n^{3} /\left(4 d^{s}\right)$ and we can use this to compute the right $p$-value.

EXAmple 2. Consider the small MIXMAX- $(m, k, d, c)$ proposed in the MIXMAX implementation of Savvidy (2017), for which $m=2^{61}-1, k=8, d=0$, and $c=2^{53}+1$. If we take all $k$ coordinates of each vector, Proposition 1 tells us that for $I=\{1,8,9\}$, all the points of $\Psi_{3}(I)$ are in only two parallel planes, and this is also true for any set $I^{\prime}$ that contains $I$. For this particular $I$, this gives $S_{3}(I)=6.69 \times 10^{-19}=M_{10}$, and therefore $M_{s^{\prime}} \leq 6.69 \times 10^{-19}$ for all $s^{\prime} \geq 10$. This value is much smaller than the worst measure we were able to find by random search in Example 1. If we insist on taking only subsets of successive coordinates starting at 0 , i.e., $I=\{0,1, \ldots, s-1\}$, we find $d_{s}=1 / 3$ with $S_{s} \approx 1.009 \times 10^{-16}$ for $s=9$, and $d_{s}=1 / \sqrt{3}$ with $S_{s} \approx 2.463 \times 10^{-15}$ for $s=10$, which are again very small. We checked that $d_{s}=1 / \sqrt{3}$ for $s \geq 10$ up to at least $s=48$.

We applied the collision test in $s=16$ dimensions, with $d=8, n=4 \times 10^{7}$, and $N=10$. The expected total number of collisions was about 28 and we observed $C_{\text {tot }}=314$. This gives the $p$-value $p \approx 6 \times 10^{-206}$. The birthday spacings test with $s=16, d=16, n=3 \times 10^{7}$, and $N=10$, gave a $p$-value smaller than $p<10^{-300}$. We also tried a collision test in $s=6$ dimensions by taking only the first 3 values of each block of 8 , with $d=128, n=4 \times 10^{7}$, and $N=10$. We expected about 1,818 collisions and we observed 116,218; this gives $p<10^{-300}$. We see that these simple tests easily detect the bad structure.

To avoid the structure given in Proposition 1, we may skip one (or both) of the two coordinates involved in this linear relation. But Propositions 4 and 6 still apply, even if we skip the first three coordinates of each vector. Given the large value of $c$, the matrix $\mathbf{A}$ has many large entries, so one might have hoped for a good lattice structure if we skip some coordinates. But here, $m=256 c-257$, and Proposition 6 applies with $q=256$ and $r=-257$.

The first part of the proposition (with $j=0$ ) says for example that for $I=\{4,5,11,12,13\}$, $\Psi_{s}(I)$ is contained in at most $5 q+|q+r|-1=1280$ equidistant parallel hyperplanes at distance $1 / \ell$ apart, where $\ell^{2}=7 q^{2}+(q+r)^{2}=458753$, i.e., $\ell \approx 677.31$, for $s=5$. By applying the spectral test numerically, we found that this $\ell$ is also the exact length $\ell_{s}(I)$ of the shortest vector for this particular $I$ (and also for $I^{\prime}=\{0,1, \ldots, 13\}$ ), and it gives $S_{s}(I) \approx 2.386 \times 10^{-16}$.

The second part of the proposition tells us that for $I=\{4,5,6,11,12,13,14\}$, the points of $\Psi_{s}(I)$ are also all covered by another family of equidistant parallel hyperplanes at distance $1 / \ell$ apart, where $\ell^{2}=5 q^{2}+r^{2}+(q+r)^{2}=393730$, i.e., $\ell \approx 627.48$, in $s=7$ dimensions. This $\ell$ is also the exact length $\ell_{s}(I)$ of the shortest vector for this $I$. The corresponding normalized figure of merit is $S_{s}(I) \approx 2.022 \times 10^{-16}$, again a much smaller value than the worst we could find in Example 1. 
To see if simple tests can detect this structure, we implemented a version of the generator that skips the first 3 values and keeps the next 5 , in each block of 8 , and we applied the birthday spacings test in $s=10$ dimensions with $d=64, n=10^{7}$, and $N=10$. The expected number of collisions was 2168 and we observed $C_{\text {tot }}=4220$. This gives a $p$-value smaller than $10^{-300}$.

EXAMPLE 3. We now look at the larger MIXMAX- $(m, k, d, c)$ with $m=2^{61}-1, k=17$, $d=0$, and $c=2^{36}+1$, proposed by Savvidy (2017) and widely adopted in software. For $I=\{1,17,18\}$ and any superset of this $I$, all the points of $\Psi_{3}(I)$ are in two parallel planes at distance $1 / \sqrt{3}$. With subsets of successive coordinates starting at 0 , we find $d_{s}=1 / \sqrt{18}$ with $S_{s} \approx 1.115 \times 10^{-17}$ for $s=18$, and $d_{s}=1 / \sqrt{3}$ with $S_{s} \approx 3.656 \times 10^{-17}$ for $s=19$, which are again very small.

Here $m=2^{25} c-2^{25}-1$, so $q=2^{25}$ and $r=-2^{25}-1$ in Proposition 6 , which yields a dual vector of length $\ell=\left(7 \times 2^{50}+1\right)^{1 / 2} \approx 8.878 \times 10^{7}$ for $I=\{4,5,11,12,13\}$ and gives $\Psi_{5}(I)=$ $3.127 \times 10^{-11}$. With $I=\{4,5,6,11,12,13,14\}$, we get a dual vector of length $\ell \approx 8.219 \times 10^{7}$, which gives $S_{7}(I) \approx 2.648 \times 10^{-11}$.

We applied the birthday spacings test to this generator with $s=20, d=8, n=10^{7}$, and $N=10$. Each block of 20 successive output values was formed by taking all 17 values of one block and the first three values of the next block, and the remaining values of the second block were not used. The expected number of collisions was 2168.4 and we observed 5863, for a $p$-value smaller than $10^{-300}$.

EXAmple 4. This MIXMAX- $(m, k, d, c)$ example was also proposed (and highly recommended) by Savvidy (2017). It has $m=2^{61}-1, k=240, d=487013230256099140$, and $c=2^{51}+1$. Proposition 1 tells us that for $I=\{1,240,241\}$, all the points of $\Psi_{3}(I)$ are in only two parallel planes and similarly for any set $I^{\prime}$ that contains $I$.

As in the previous example, some bad projections appear even if we skip the first three coordinates of each output vector. Here we have $m=1024 c-1025$, so Proposition 6 applies with $q=1024$ and $r=-1025$. The proposition tells us that for $I=\{4,5,243,244,245\}, \Psi_{s}(I)$ is contained in at most $5 q+|q+r|-1=5120$ equidistant parallel hyperplanes at distance $1 / \ell$ apart, where $\ell^{2}=7 q^{2}+(q+r)^{2}=7340033$, i.e., $\ell \approx 2709.245$, for $s=5$. This $\ell$ turns out to be the exact length of the shortest vector for this $I$. The corresponding normalized figure of merit is $S_{s}(I) \approx 9.5436 \times 10^{-16}$.

For the larger index set $I=\{4,5,6,243,244,245,246\}$, the points of $\Psi_{s}(I)$ all belong to another family of parallel hyperplanes at distance $1 / \ell$ apart, where $\ell^{2}=5 q^{2}+r^{2}+(q+r)^{2}=$ 6293506 , i.e., $\ell \approx 2508.686$, in $s=7$ dimensions. This $\ell$ is again the length of the shortest vector for this $I$ and we have $S_{s}(I) \approx 8.0836 \times 10^{-16}$, which is again very small. This means that the distance between successive hyperplanes that contain all the points is much larger than what one should expect with $m=2^{61}-1$ in 7 dimensions. Note that changing $d$ would have no impact on these results, because $d$ does not appear in the bounds. 


\section{Conclusion}

We have examined the lattice structure of the vectors (or points) of successive output values produced by the MIXMAX generator. We showed that the projections of those points over certain subsets of coordinates have bad structures, in which all the points belong to a small number of parallel hyperplanes, much smaller than what one would expect given the size of the modulus. In particular, for a very large number of projections (all those that contain three specific indices), all the points are in only two hyperplanes. One can get rid of these bad structures by skipping some output values, but we saw that other structures then show up, due to linear relationships between other subsets of coordinates. These relationships come from the special structure of the matrix A. One can alleviate this weak behavior by skipping more coordinates in the output vectors (those that are involved in the bad linear relationships), but this would slow down the generator.

What is the practical impact of these bad structures on simulation results if we do not skip coordinates, or if we skip just a few? The results of simple empirical tests given here provide a partial answer: the tests easily detect the structure. For most applications there may be no visible impact, but for some applications the poor structures may introduce significant bias if there is some kind of alignment or synergistic effect between the poor lattice structure and the way the random numbers are used in the application, as exemplified in our statistical tests. This type of effect is not easy to predict in general, but it has been observed in the past for RNGs that have a poor lattice structure similar to the one that we have unveiled here; see Ferrenberg et al. (1992), Tezuka et al. (1993), and L'Ecuyer (1997), for example. Therefore, it is important for the MIXMAX users to be aware of these structural properties and be cautious about them.

\section{Acknowledgments}

This work has been supported by an NSERC-Canada Discovery Grant, a Canada Research Chair, and an Inria International Chair to P. L'Ecuyer. Marc-Antoine Savard helped doing spectral test computations and Théo Guillaumot ran the statistical tests. P. L'Ecuyer wants to thank G. K. Savvidy for inviting him to the MIXMAX Network Meeting at the CERN, in Geneva, in July 2016, which triggered his interest in the MIXMAX generator. We thank the reviewers, the Associate Editor, and the Area Editor B. Tuffin, whose comments helped improving the paper.

\section{References}

Afflerbach L, Grothe H (1988) The lattice structure of pseudo-random vectors generated by matrix generators. Journal of Computational and Applied Mathematics 23:127-131.

Akopov NZ, Savvidy GK, Savvidy NGTA (1991) Matrix generators for pseudorandom numbers. Journal of Computational Physics 97:573-579. 
Conway JH, Sloane NJA (1999) Sphere Packings, Lattices and Groups. Grundlehren der Mathematischen Wissenschaften 290 (New York: Springer-Verlag), 3rd edition.

Couture R, L'Ecuyer P (1994) On the lattice structure of certain linear congruential sequences related to AWC/SWB generators. Mathematics of Computation 62(206):798-808.

Couture R, L'Ecuyer P (1996) Orbits and lattices for linear random number generators with composite moduli. Mathematics of Computation 65(213):189-201.

Coveyou RR, MacPherson RD (1967) Fourier analysis of uniform random number generators. Journal of the ACM 14:100-119.

Ferrenberg AM, Landau DP, Wong YJ (1992) Monte Carlo simulations: Hidden errors from "good" random number generators. Physical Review Letters 69(23):3382-3384.

Fincke U, Pohst M (1985) Improved methods for calculating vectors of short length in a lattice, including a complexity analysis. Mathematics of Computation 44:463-471.

Grothe H (1988) Matrixgeneratoren zur Erzeugung Gleichverteilter Pseudozufallsvektoren. Dissertation (thesis), Tech. Hochschule Darmstadt, Germany.

James F (1994) RANLUX: A Fortran implementation of the high-quality pseudorandom number generator of Lüscher. Computer Physics Communications 79:111-114.

Knuth DE (1998) The Art of Computer Programming, Volume 2: Seminumerical Algorithms (Reading, MA: Addison-Wesley), third edition.

L'Ecuyer P (1990) Random numbers for simulation. Communications of the ACM 33(10):85-97.

L'Ecuyer P (1994) Uniform random number generation. Annals of Operations Research 53:77-120.

L'Ecuyer P (1997) Bad lattice structures for vectors of non-successive values produced by some linear recurrences. INFORMS Journal on Computing 9(1):57-60.

L'Ecuyer P (1999) Tables of linear congruential generators of different sizes and good lattice structure. Mathematics of Computation 68(225):249-260, see the Errata at http://www .iro. umontreal.ca/ lecuyer/ myftp/papers/latrules99Errata.pdf.

L'Ecuyer P (2006) Uniform random number generation. Henderson SG, Nelson BL, eds., Simulation, 55-81, Handbooks in Operations Research and Management Science (Amsterdam, The Netherlands: Elsevier), chapter 3 .

L'Ecuyer P (2012) Random number generation. Gentle JE, Haerdle W, Mori Y, eds., Handbook of Computational Statistics, 35-71 (Berlin: Springer-Verlag), second edition.

L'Ecuyer P (2017) History of uniform random number generation. Proceedings of the 2017 Winter Simulation Conference, 202-230 (IEEE Press).

L'Ecuyer P, Couture R (1997) An implementation of the lattice and spectral tests for multiple recursive linear random number generators. INFORMS Journal on Computing 9(2):206-217. 
L'Ecuyer P, Munger D, Oreshkin B, Simard R (2017) Random numbers for parallel computers: Requirements and methods, with emphasis on GPUs. Mathematics and Computers in Simulation 135:3-17, open access at http://dx.doi.org/10.1016/j.matcom.2016.05.005.

L'Ecuyer P, Simard R (2001) On the performance of birthday spacings tests for certain families of random number generators. Mathematics and Computers in Simulation 55(1-3):131-137.

L'Ecuyer P, Simard R (2007) TestU01: A C library for empirical testing of random number generators. ACM Transactions on Mathematical Software 33(4):Article 22.

L'Ecuyer P, Simard R (2014) On the lattice structure of a special class of multiple recursive random number generators. INFORMS Journal on Computing 26(2):449-460.

L'Ecuyer P, Simard R, Wegenkittl S (2002) Sparse serial tests of uniformity for random number generators. SIAM Journal on Scientific Computing 24(2):652-668.

L'Ecuyer P, Touzin R (2004) On the Deng-Lin random number generators and related methods. Statistics and Computing 14:5-9.

Lüscher M (1994) A portable high-quality random number generator for lattice field theory simulations. Computer Physics Communications 79:100-110.

Marsaglia G (1968) Random numbers fall mainly in the planes. Proceedings of the National Academy of Sciences of the United States of America 60:25-28.

Marsaglia G, Zaman A (1991) A new class of random number generators. The Annals of Applied Probability $1: 462-480$.

Niederreiter H (1986) A pseudorandom vector generator based on finite field arithmetic. Mathematica Japonica 31:759-774.

Savvidy GK, Ter-Arutyuntan-Savvidy NG (1991) On the Monte Carlo simulation of physical systems. Journal of Computational Physics 97:566-572.

Savvidy KG (2015) The MIXMAX random number generator. Computer Physics Communications 196:161165.

Savvidy KG (2017) MIXMAX Manual. See https://www.hepforge.org/archive/mixmax/MANUAL.pdf.

Savvidy KG, Savvidy GK (2016) Spectrum and entropy of C-systems MIXMAX random number generator. Chaos, Solitons and Fractals 91:33-38.

Tahmi EHADE (1982) Contribution aux Générateurs de Valeurs Aléatoires. Dissertation (thesis), Université des Sciences et Technologies Houari Boumédienne, Algeria.

Tezuka S, L'Ecuyer P (1992) An analysis of add-with-carry and subtract-with-borrow generators. Proceedings of the 1992 Winter Simulation Conference, 443-447 (IEEE Press).

Tezuka S, L'Ecuyer P, Couture R (1993) On the add-with-carry and subtract-with-borrow random number generators. ACM Transactions of Modeling and Computer Simulation 3(4):315-331. 